\title{
Introduction: applying a landscape perspective to digital cultural heritage
}

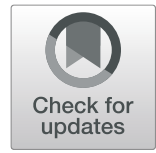

Chen Yang ${ }^{1 *}$ and Kelly Greenop ${ }^{2}$

Technology has always existed to mediate understanding of places and artefacts, from the invention of carving tools to make images of the game being hunted or sacred objects within ancient societies, to the digitisation of places that new technologies have enabled in recent decades. The impetus to interpret the world and using tools to do so is ageold but has new connotations in the current era of Industry 4.0. We are not only digitising, but also connecting places, objects and communities virtually, and augmenting interpretation of heritage places through digital means to better understand and communicate their values, and to reveal values of digitally created artefacts. Digital cultural heritage is a relatively new discipline which seeks to combine the inherently conservative nature of heritage with contemporary digital technologies, which are experimental, evolving and challenging, even disruptive, to the status quo across many disciplines, including heritage. Nevertheless, what may seem an unlikely pairing in fact opens new possibilities for the practice and conceptualisation of heritage. The overarching goal of digital cultural heritage is to improve how the recording, interpretation and storage of heritage information are conducted making it more detailed, complete, sustainable and accessible, and to develop new ways of imagining heritage and its relationship to societies.

The challenge for this new discipline is to imagine a future for heritage, where conservation of not only heritage places but the traditions of the heritage discipline itself which are deeply treasured by many, adapts to new opportunities. This entails development of accessible and useful technologies to meet the needs of heritage practitioners, asset owners, resident- and non-resident heritage communities, and a diverse set of publics. Digital cultural heritage aims to be able to address heritage needs at world and national levels of significance as well to develop techniques and approaches for less officially recognised heritage settings with scant or no

\footnotetext{
* Correspondence: chen.yang@tongji.edu.cn

${ }^{1}$ Department of Landscape Architecture, College of Architecture and Urban Planning, Tongji University, 1239 Siping Road, Yangpu District, Shanghai 200092, China

Full list of author information is available at the end of the article
}

funding. Furthermore, it offers possibilities for addressing some of the ongoing open questions within heritage, for example, how to identify and engage broader communities in heritage; how to record and share intangible cultural heritage and the changeable 'living heritage' which is developing every day; and how to safeguard and make accessible both born-digital heritage and digital records of tangible heritage places into the future.

The technical innovations that comprise new digital cultural heritage techniques are being advanced by experts in their chosen technologies, but these innovations do not necessarily address heritage-focussed disciplinary questions, and sometimes emphasise technical questions in isolation from the end-users or purported beneficiaries of the technology. Heritage practitioners and stakeholders must, at a minimum, develop technical literacy in the digital options available, and ideally become partners with technicians to decide the digital future of heritage. Current decisions in this realm will determine how heritage is recorded, presented, viewed and archived for coming generations. We advocate for a critical digital cultural heritage in which an investigation of not only how to digitise places and events or to digitally create heritage artefacts, but why this ought to be done. The role of researchers is to interrogate these digitisation efforts, and speculate what they may mean for changing concepts of heritage as a result.

Key questions faced by digital cultural heritage in its current moment are: why ought a place be digitised, and what might be the benefits in doing this? What new opportunities does digitisation bring and what questions does it raise? Whose voices, stories and perspectives are being represented, or excluded, and why? What are the boundaries of these digital technologies for heritage purposes, that is, what can we not represent yet, or perhaps ever? Are there risks to digitisation and indeed some cases where it should not be conducted? What professional disciplines are leading digital cultural heritage and why, and how does this affect outcomes? And finally, how can digital cultural heritage be used for good? Digital technologies offer a chance to craft a new or better view or one from a different perspective and therefore offer an 
opportunity to understand heritage places and events in novel and divergent ways.

Adding the specific focus of a landscape perspective to this set of concerns brings the focus to a scale where digital technologies can address existing problems of representation and comprehensiveness. Over the past three decades, the concept of 'landscape' has succeeded both as a cultural heritage category and as a methodology of heritage conservation. The number of World Heritage Cultural Landscapes has grown from 30 in 2002 to 112 by 2019, becoming one of the most active categories within World Heritage. At the same time, the landscape perspective has also expanded our understanding of cultural heritage, so that it includes not only elite, magnificent, and static sites, but also vernacular and dynamic places. The landscape perspective also shifts the developing digital cultural heritage orthodoxy beyond the architecture or object-focussed approach to encompass the broader scale of towns, villages, natural landscapes and cultural routes. These categories and domains have helped to forge a more inclusive heritage conservation framework, which has greatly contributed to cultural diversity, sustainable development, and social democracy, goals also shared by digital cultural heritage projects.

The landscape perspective raises new requirements on cultural heritage conservation methods and technologies. It regards nature-culture interactions as the focus when evaluating heritage values, and emphasises the deciphering of the relationship between people and the environment. The sustainable use of nature by humans and the biocultural diversity information they generate need to be effectively identified and recorded. This view incorporates natural elements and their evolution into the cultural heritage conservation realm, which puts forward higher requirements for the efficiency, accuracy and comprehensiveness of technologies for heritage information acquisition. Furthermore, the landscape perspective maintains that heritage cannot be viewed in isolation, and it must be integrated into the overall social, political, economic, and cultural context to locate and interpret it. This means that heritage conservation requires the integration of diverse information, including historical, tangible, intangible, natural, and cultural features. Digital technologies are key to supporting such efficient data management platforms. More importantly, the concept of landscape highlights the 'way of seeing' heritage, that is, being conscious of interpreting the meanings and values of cultural heritage, and assessing authenticity and integrity from a specific cultural perspective. In this process, the dissemination and sharing of heritage knowledge are essential. Contemporary digital tools will promote effective heritage interpretation, provide more support for the transmission of voices from different cultural communities, and achieve appropriate understanding and conservation strategies.

Although the landscape perspective has been widely accepted by the cultural heritage academic community, it has still not been fully realised in the practice of cultural heritage conservation and management. This is especially evident in the lack of operating methods and technical guidelines. At many cultural heritage sites, the recording and monitoring of physical evidence still mainly consists of 'cultural relics protection'. Some factors affecting how landscapes are recorded, interpreted and valued at cultural heritage sites include: a large amount of information on natural and intangible heritage elements cannot be taken into consideration in heritage decision-making due to lack of necessary technologies; fragmentation of heritage information is still one of the main obstacles to efficient protection; paper archives and the corporate memories held by site managers are still important ways to support heritage management, but they may not be reliable and easy to replicate for others; public participation still relies on specific projects as the gateway for the public, and longterm mechanisms and platforms for heritage commentary and public participation have not been widely established. With all these factors in mind, there is still a long way to go from 'a landscape perspective' to 'a landscape approach' in cultural heritage practice.

The development of digital technologies in recent years has provided important opportunities for bridging the perspective and the approach, and have gradually formed a new field of digital heritage landscape research. New surveying technologies exemplified by digital closerange photogrammetry, LiDAR, and drone tilt photography technologies have greatly improved the efficiency of geographical digital mapping, and the identification and dynamic monitoring of a cultural heritage place have become possible. The spatial data management technologies of geographical information systems (GIS) are increasingly developed, and more powerful threedimensional spatial analysis, multi-information integration, and open data frame functions provide important opportunities for the digital management of cultural heritage information and the realisation of multidimensional panoramic simulation of heritage. Further to this, interactive multimedia represented by mixed reality tools and public interpretation technology will greatly improve the readability and attractiveness of heritage interpretation. At the same time, crowdsourced data will greatly expand the scope and approach of multi-value identification of heritage. Therefore, digital technologies will provide important support for the application of a landscape perspective in cultural heritage conservation, and bring important changes to the conservation workflow and conceptualising what landscape heritage is.

This special issue 'Applying a Landscape Perspective to Digital Cultural Heritage', reflects selected papers presented at the 'digital cultural heritage: FUTURE VISIONS, 
a landscape perspective' Conference, held in the College of Architecture and Urban Planning at Tongji University Shanghai on the 23rd and 24th of November 2019. This conference was co-organised by Tongji University, The University of Queensland and supported by the ICOMOS-IFLA International Scientific Committee on Cultural Landscapes (ISCCL). The conference shifted the perspective from digital cultural heritage focussed mainly on architectural settings to foreground the landscape scale and the challenges specific to heritage landscapes, to examine how digital cultural heritage within landscape architecture is being addressed both technically and theoretically. An article reporting on the conference themes including the keynote speeches is featured in this issue, by Associate Professor Chris Landorf from The University of Queensland. Conference papers addressing some key disciplinary issues were selected and further developed into articles for this special issue.

Mario Santana Quintero and Luigi Barazetti first discuss some of the fundamental issues of digital documentation of cultural heritage. They point out the revolutionary changes brought about by new digital technologies in cultural heritage recording and archival work and introduce a deeper discussion on the code of conduct and the ethical issues of heritage practices. They propose that the new data medium improves the efficiency of heritage research and practice, and brings new challenges, including data separation due to technological diversity, excess data and its quality control and storage, its future use, etc. Based on this, the article establishes an ethical framework for digital documentation of cultural heritage and sets up professional behaviour standards, responsibilities, professional practices, and protection of the interests of the public to ensure more appropriate and effective heritage resource management. The article demonstrates the use of this ethical framework through the digital recording project of the Nea Paphos World Heritage site in Cyprus.

Sarah Karle and Richard Carman's article focuses on rural landscapes in the United States and explores how digital tools can be used to construct large-scale mapping methods for the study and protection of landscape heritage. They explain how to use a Historical Geographic Information System (HGIS) methods to enhance the accessibility of landscape archives and to facilitate public participation. The dynamic plant material and easily modified spatial conditions in the landscape are difficulties often faced by traditional protection methods. In the Prairie States Forestry Project, the authors constructed a digital approach to reduce the workload of field investigations and spatial analysis in large-scale heritage research. This article demonstrates the important contribution of digital technology in representing the historical dimensions of cultural landscapes and dynamic landscape features, highlighting the application of
GIS into the management and representation of historical information of heritage sites, which has important reference value for other heritage sites.

Chen Yang and Feng Han provide an overview of a comprehensive databasing project that beings to explore the pragmatic uses of digital databases for recording and managing landscape-scale cultural heritage sites. They describe the production of a geo-database for the Slender West Lake landscape cultural heritage area, and the inclusion of both tangible and intangible heritage values in this database. They develop a framework of how such a database should be built, including practical guidance on GIS and other technical considerations for heritage landscape-specific use, with an emphasis how to integrate different information from a variety of sources and featuring diverse sets of information is explored. There was a specific focus on how to capture and locate intangible landscape elements using this framework. Slender West Lake cultural landscape was used as a case study, providing an important benchmark for testing this approach and its further development at other landscape heritage sites in China and beyond.

Stanislav Roudavski and Julian Rutten's article addresses the issue of landscape heritage in a more-thanhuman context, that of the heritage of trees as both habitat and heritage. They make a case for the pursuit of nonhuman heritage and make claims for a concept of animals having cultures which are supported by and occur within habitats that humans also value, but perhaps for different reasons. When examining arboreal habitats from the nonhuman perspective Roudavski and Rutten analyse and record the characteristics of trees and how they are used by animals, and in doing so reveal the technical and ethical challenges in recording such nonhuman heritage. The landscape perspective and the elements that comprise it-in this case trees and their hollows as habitat-demonstrate the importance of new theories of heritage where we consider more-thanhuman needs and the values we place on them.

2020 will be the first year that $5 \mathrm{G}$ mobile communication technology enters commercial use, which may bring about the seventh information revolution in human society. Undoubtedly, with the high-speed and low-latency information transmission, we will have a new technical foundation for the digital capture of cultural heritage and entire living and ecological environments. The continuous emergence of new technologies will extend our perception capacity and help deepen our understanding of the outside world. Although the speed of technological development is sometimes unfathomable, and intelligent learning makes it possible for machines to surpass the human brain in terms of information processing, it is nevertheless difficult for technologies to solve some fundamental problems, including defining 
heritage value, ownership, authenticity, integrity, etc. These remain in the human sphere and rightly so, with technologies being a tool to help us identify, maintain and connect with heritage. How to use these technologies to deepen our understanding of heritage, improve the efficiency of heritage management, and achieve the sustainable transmission of heritage information to future generations are the pressing questions that researchers and practitioners need to work on together, within the changing technological context. The first issue of Built Heritage in 2020, focusing on the frontier of cultural heritage conservation, will promote the debate on this theme and make important contributions to the theory and practice of cultural heritage conservation. We hope to revisit these themes in future years and reflect on the positive contributions that the digital has made to cultural heritage in the intervening period.

\section{Acknowledgements}

Not applicable.

\section{Authors' contributions}

Both authors read and approved the final manuscript.

\section{Funding}

Not applicable.

Availability of data and materials

Not applicable.

\section{Competing interests}

The authors declare that they have no competing interests.

\section{Author details}

${ }^{1}$ Department of Landscape Architecture, College of Architecture and Urban Planning, Tongji University, 1239 Siping Road, Yangpu District, Shanghai 200092, China. ${ }^{2}$ School of Architecture, The University of Queensland, Brisbane, St Lucia QLD 4072, Australia.

Received: 6 February 2020 Accepted: 9 February 2020

Published online: 25 March 2020

\section{Publisher's Note}

Springer Nature remains neutral with regard to jurisdictional claims in published maps and institutional affiliations.

\section{Submit your manuscript to a SpringerOpen ${ }^{\mathcal{O}}$ journal and benefit from:}

- Convenient online submission

- Rigorous peer review

- Open access: articles freely available online

- High visibility within the field

- Retaining the copyright to your article 